\title{
Revised Distributional Forms of the Laplacian and Poisson's Equation, Their Implications, and All Related Generalizations
}

\author{
ALP KUSTEPELI ${ }^{1}$ \\ ${ }^{1}$ Department of Electrical and Electronics Engineering, Izmir Institute of \\ Technology, Izmir, Turkey
}

\begin{abstract}
The theory of distributions of L. Schwartz is a very useful and convenient way for the analysis of physical problems since physical distributions, especially charge distributions yielding the discontinuity of the potential and boundary conditions, can be correctly described in terms of mathematical distributions. To obtain the charge distributions, the distributional form of the Laplacian is applied to the Poisson's equation; therefore, for the correct representations and interpretations, the distributional forms and their proper applications are very important. In this article, it is shown that the distributional form of the Laplacian has been presented by Schwartz and also others with a missing term, leading to confusing and wrong results mathematically, and as a result electromagnetically; and the revised, correct, and complete distributional representations of the Laplace operator, the Poisson equation, and double layers, defined as the dipole layer and equidensity layer, are obtained and presented with detailed discussions and explanations including boundary conditions. By using the revised form of the Laplacian, Green's theorem is obtained explicitly with special emphases about important points and differences with previous works. The generalized forms of the Laplacian, Poisson's equation, charge densities, boundary conditions, and Green's theorem are also presented when there is a multi-layer on the surface of discontinuity.
\end{abstract}

Keywords distribution theory, discontinuities, electromagnetism, charge densities, boundary conditions, multi-layers

\section{Introduction}

The variation of potential function $V$ across a surface $S$ is important since it determines the charge density on $S$. It is known that if $V$ is continuous and its derivative is discontinuous, a surface charge density representing a single layer occurs on $S$. It is also known that $V$ must be discontinuous across $S$ to form a double layer on the surface. The discontinuity of potential was investigated by Maxwell (1954), and his extraordinary approach, supposing that the points in the surface of discontinuity belong to both regions, can be seen in Article 78 of his treatise. If one also considers the footnote given by Thomson in Article 102c of Maxwell's treatise about the charge densities on two very closely spaced surfaces, where their potential difference can be treated as a discontinuity in the potential function, it can

Received 20 November 2014; accepted 16 April 2015.

Address correspondence to Alp Kustepeli, Department of Electrical and Electronics Engineering, Izmir Institute of Technology, Gulbahce Koyu, 35430 Urla, Izmir, Turkey. E-mail: alpkustepeli@iyte.edu.tr 
be seen that the charge densities are related not only to the potential difference but also to the geometric properties of the surfaces, e.g., the first curvatures of the surfaces.

The importance of the geometric properties of the surface was also emphasized by Jackson (1975, p. 51), which was taken into consideration by Pappas (1986). Kanwal (1988) pointed out in a note about (Pappas 1986) that the jumps of functions and their derivatives across the surfaces of discontinuity can be obtained in terms of the geometric quantities by using distributional approach and considering a single layer potential for that special case.

Since physical distributions can be correctly described in terms of mathematical distributions (Schwartz, 2008, p. 77; Van Bladel, 1995, p. 18), the distribution theory is very convenient for the examination of those kinds of physical problems, and immediately after its introduction by Schwartz (1950), a very early distributional treatment of classical electrodynamics was presented in detail by Taylor (1956, 1958), considering Maxwell equations as distribution equations, fields and potentials as distributions, and the Dirac delta distribution in four-dimensional space-time.

Other very valuable works and information about the application of distributions in the electromagnetic field theory can be seen in Gagnon (1970), Rădulet and Ciric (1971), İdemen (1973, 1990, 2011), and Polat (2005, 2006). Kanwal (1988) additionally mentioned in a note that the jump relations for a double layer were also presented in Kanwal (1983, p. 255). Since Van Bladel (1995, p. 206) described two methods of a double-layer formation, one resulting in a dipole layer and the other leading to an equidensity double layer, how those two different double layers can be obtained from the discontinuity of the potential function $V$, their exactly correct volume density representations, and their boundary relations are important. Therefore, this study intends to make a detailed investigation of the distributional representations for and related to the Laplacian, Poisson's equation, charge densities, and boundary conditions to obtain their accurate forms and, for their generalized forms, to obtain the most general expressions when there is a multi-layer on the discontinuity surface.

\section{Revised Distributional Form of the Laplacian and Green's Theorem}

The distributional forms of gradient, divergence, and curl can be obtained as (Gagnon, 1970; Rădulet \& Ciric, 1971; İdemen, 1973; Van Bladel, 1999)

$$
\begin{gathered}
\nabla V=\{\nabla V\}+\hat{n} \llbracket V \rrbracket \delta(S), \\
\nabla \cdot \vec{A}=\{\nabla \cdot \vec{A}\}+\hat{n} \cdot \llbracket \vec{A} \rrbracket \delta(S), \\
\nabla \times \vec{A}=\{\nabla \times \vec{A}\}+\hat{n} \times \llbracket \vec{A} \rrbracket \delta(S),
\end{gathered}
$$

where $S$ is the surface of discontinuity, which separates the two contiguous regions, $\vartheta_{1}$ and $\vartheta_{2}$, where $\vec{r} \notin S$. If a scalar function considered in those regions, represented as $V=\{V\}$ and \{\} , later called the regular part of the quantity considered, its jump across $S$ is denoted by $\llbracket V \rrbracket=V^{2}-V^{1}$ in Eq. (1), where $V^{2}$ and $V^{1}$ are the boundary values of $V$ on $S$ from $\vartheta_{2}$ and $\vartheta_{1}$, respectively. The unit normal vector of the surface is $\hat{n}$, and it is directed into $\vartheta_{2} . \delta(S)$ denotes the Dirac delta distribution concentrated on $S$. The representations in 
Eqs. (2) and (3) are similar for vector function $\vec{A}$. The surface and those regions can be described by using a characteristic function $w(x, y, z)$, as presented by Maxwell (1954) in Article 78 of his treatise and also as given by Namias (1977) and İdemen (2011, p. 53), such that $w=0, w<0$, and $w>0$ determine $S, \vartheta_{1}$, and $\vartheta_{2}$, respectively. In this case, one can also use a similar distribution $\delta(w)$ instead of $\delta(S)$, and Eqs. (1)-(3) can be modified by using the relation $\delta(S)=|\nabla w| \delta(w)$ (Namias, 1977; İdemen, 2011, p. 53). In addition to Eqs. (1)-(3), many other useful distributional relations can be found in Gagnon (1970). The Laplacian, denoted by $\Delta$, can be obtained by taking the divergence of Eq. (1), using Eq. (2), as

$$
\begin{aligned}
\Delta V & =\nabla \cdot(\nabla V) \\
& =\nabla \cdot(\{\nabla V\}+\hat{n} \llbracket V \rrbracket \delta(S)) \\
& =\nabla \cdot\{\nabla V\}+\nabla \cdot(\hat{n} \llbracket V \rrbracket \delta(S)) \\
& =\{\Delta V\}+\hat{n} \cdot \llbracket \nabla V \rrbracket \delta(S)+\nabla \cdot(\hat{n} \llbracket V \rrbracket \delta(S)) \\
& =\{\Delta V\}+\llbracket \llbracket \frac{\partial V}{\partial n} \rrbracket \delta(S)+\nabla \cdot(\hat{n} \llbracket V \rrbracket \delta(S)),
\end{aligned}
$$

which is also the result given and used by Gagnon (1970), who presented a very elegant way of obtaining Green's theorem or Green's second identity by using the last line of Eq. (4) in the formulation

$$
\langle\Delta f, t\rangle=\langle f, \Delta t\rangle
$$

and \langle\rangle is defined by

$$
\langle f, t\rangle=\int f t d \vartheta
$$

where $f$ is a distribution, $t$ is a test function, and the integral sign without the limits indicates that the integration is extended over all space (Schwartz, 2008, p. 77; Gagnon, 1970; Kanwal, 1983, p. 26; Van Bladel, 1995, p. 6; 2007, p. 1090). Instead of taking the divergence of the last term, Gagnon transferred the differentiation to the test function with the use of

$$
\langle\nabla \cdot \vec{F}, t\rangle=-\langle\vec{F}, \nabla t\rangle=-\int \vec{F} \cdot \nabla t d \vartheta
$$

where $\vec{F}$ is a vector distribution. To obtain an explicit form of the Laplacian, more attention must be paid to that last term of the last line in Eq. (4) and the divergence taken, by considering the identities for scalar and vector distributions given in the appendix of Gagnon (1970) as

$$
\nabla \cdot(\hat{n} \llbracket V \rrbracket \delta(S))=\llbracket V \rrbracket \delta(S) \nabla \cdot \hat{n}+\nabla(\llbracket V \rrbracket \delta(S)) \cdot \hat{n},
$$

where $\nabla \cdot \hat{n}=-J$ and $J=1 / R_{1}+1 / R_{2}$ is the first curvature of the surface of discontinuity $S$ with $R_{1}$ and $R_{2}$ being the two principal radii of curvature (Van Bladel, 2007, p. 1026). The last term in Eq. (8) is 


$$
\begin{aligned}
\nabla(\llbracket V \rrbracket \delta(S)) \cdot \hat{n} & =(\nabla \llbracket V \rrbracket \delta(S)+\llbracket V \rrbracket \nabla \delta(S)) \cdot \hat{n} \\
& =\nabla \llbracket V \rrbracket \cdot \hat{n} \delta(S)+\llbracket V \rrbracket \nabla \delta(S) \cdot \hat{n}
\end{aligned}
$$

and since $\llbracket V \rrbracket$ may only be a function of surface coordinates in the Dupin coordinate system (Van Bladel, 2007, p. 1027; Tai, 1992, p. 15), $\nabla \llbracket V \rrbracket$ has only tangential components and $\nabla \llbracket V \rrbracket \cdot \hat{n}=\nabla_{S} \llbracket V \rrbracket \cdot \hat{n}=0$, where $\nabla_{S}$ represents the surface gradient given by Van Bladel (2007, p. 1027). Tai (1992, p. 80) used another approach and presented a different representation for the surface gradient. This study uses Van Bladel's treatment, which is the classical approach for the surface operators given by Weatherburn (1955). Considering $\nabla \delta(S) \cdot \hat{n}=\partial \delta(S) / \partial n$, or in general, $\nabla \delta^{(k)}(S)=\hat{n} \delta^{(k+1)}(S)$, which can be obtained by combining the Cartesian derivatives presented in Eq. (5.4.8) of Kanwal (1983, p. 117), where $\delta^{(k)}(S)$ denotes the normal derivative of the Dirac delta distribution of arbitrary order $k \geq 0$, gives

$$
\nabla(\llbracket V \rrbracket \delta(S)) \cdot \hat{n}=\llbracket V \rrbracket \nabla \delta(S) \cdot \hat{n}=\llbracket V \rrbracket \frac{\partial \delta(S)}{\partial n},
$$

or, more easily,

$$
\nabla(\llbracket V \rrbracket \delta(S)) \cdot \hat{n}=\frac{\partial}{\partial n}(\llbracket V \rrbracket \delta(S))=\llbracket V \rrbracket \frac{\partial \delta(S)}{\partial n} .
$$

If all these results are gathered to rewrite Eq. (8),

$$
\begin{aligned}
\nabla \cdot(\hat{n} \llbracket V \rrbracket \delta(S)) & =-J \llbracket V \rrbracket \delta(S)+\frac{\partial}{\partial n}(\llbracket V \rrbracket \delta(S)) \\
& =-J \llbracket V \rrbracket \delta(S)+\llbracket V \rrbracket \frac{\partial \delta(S)}{\partial n},
\end{aligned}
$$

and finally, inserting Eq. (12) in Eq. (4) gives the Laplacian of $V$

$$
\begin{aligned}
\Delta V & =\{\Delta V\}+\llbracket \llbracket \frac{\partial V}{\partial n} \rrbracket \delta(S)-J \llbracket V \rrbracket \delta(S)+\frac{\partial}{\partial n}(\llbracket V \rrbracket \delta(S)) \\
& =\{\Delta V\}+\left(\llbracket \frac{\partial V}{\partial n} \rrbracket-J \llbracket V \rrbracket\right) \delta(S)+\llbracket V \rrbracket \frac{\partial \delta(S)}{\partial n} .
\end{aligned}
$$

The distributional form of the Laplacian is presented without the term $-J \llbracket V \rrbracket \delta(S)$ in Schwartz's (2008, p. 87) fundamental book about the distribution theory and in its citations, such as Van Bladel (1995, p. 21; 1999; 2007, p. 1102), Rădulet and Ciric (1971), and Vladimirov (2002, p. 33). The form of the Laplacian is very important because it determines the resulting volume charge densities in terms of the discontinuity of potential function when the distributional form of the Poisson equation is considered. One might think that the result obtained in Eq. (13) may be incorrect, but it is consistent with Eq. (5.6.8) given by Kanwal (1983, p. 124), which is obtained with a different approach. Kanwal (1983, p. 132) obtained Green's theorem in a manner similar to that given by Gagnon (1970) by defining a normal derivative operator $d_{n}$ (Kanwal 1983, p. 114), corresponding to the divergence operator seen as the last term in Eq. (4) when the results are considered and by writing the Laplacian as 


$$
\left.\left.\Delta V=\{\Delta V\}+\llbracket \frac{\partial V}{\partial n}\right]\right\rfloor \delta(S)+\llbracket V \rrbracket d_{n} \delta(S)
$$

in Eq. (5.6.8) of Kanwal (1983, p. 124). Since the normal derivative operator is $d_{n}=$ $\partial / \partial n-J$ and it is not the normal derivative, Eq. (14) is not equal to the distributional form given with the absence of $-J \llbracket V \rrbracket \delta(S)$ of Eq. (13), as presented in Schwartz (2008, p. 87), Rădulet and Ciric (1971), Van Bladel (1995, p. 21; 1999; 2007, p. 1102), and Vladimirov (2002, p. 33). Both representations presented by Gagnon (1970), as in Eq. (4), and Kanwal (1983, p. 124), as in Eq. (14), can be regarded as implicit formulations and they do not show the geometric properties of the discontinuity surface explicitly in the distributional form of the Laplacian and also in their way of obtaining Green's theorem. If one takes into consideration the explicit form of the Laplacian given in Eq. (13) and wants to obtain Green's theorem by using

$$
\left\langle\frac{\partial \delta(S)}{\partial n}, t\right\rangle=-\left\langle\delta(S), \frac{\partial t}{\partial n}\right\rangle,
$$

as given in the literature (Schwartz, 2008, p. 88; Van Bladel, 1995, p. 19; 1999; 2007, p. 1097; Vladimirov, 2002, p. 30), one might conclude that Green's theorem cannot be obtained because of the presence of the term $-J \llbracket V \rrbracket \delta(S)$ and that there is an inconsistency in the result. However, Eq. (15) is not correct, and

$$
\left\langle\frac{\partial \delta(S)}{\partial n}, t\right\rangle \neq-\left\langle\delta(S), \frac{\partial t}{\partial n}\right\rangle .
$$

With the correct form given by Kanwal (1983, p. 114) as

$$
\left\langle\frac{\partial \delta(S)}{\partial n}, t\right\rangle=-\left\langle\delta(S), \frac{\partial t}{\partial n}-J t\right\rangle,
$$

it will be shown that Green's theorem can be obtained explicitly by using only the standard definition in Eq. (6) and without the need of any other information or definition. Additionally, by the successive applications of Eq. (17), one can also have

$$
\left\langle\delta^{(k)}(S), t\right\rangle=(-1)^{k}\left\langle\delta(S),\left(\frac{\partial}{\partial n}-J\right)^{k} t\right\rangle,
$$

which will be used later for all related generalizations. In Schwartz's (2008, p. 87) fundamental book, the reason for presenting the distributional form of the Laplacian in Eq. (II.2.46) with a missing term can be explained as follows. Schwartz (2008, p. 87) obtained the distributional form of the Laplacian by using the integral result

$$
-\int_{S} \frac{\partial t}{\partial n} \llbracket V \rrbracket d S
$$

in Eq. (II.2.45) and concluded that its corresponding distribution can be represented by $\partial(\llbracket V \rrbracket \delta(S)) / \partial n$, but actually the corresponding distribution is $\partial(\llbracket V \rrbracket \delta(S)) /$ $\partial n-J \llbracket V \rrbracket \delta(S)$, as can be seen from Eqs. (16) and (17). Therefore, the distributional 
form of the Laplacian presented by Schwartz (2008, p. 87) in Eq. (II.2.46) and by also others must be revised, and its correct form must be used as given in Eq. (13). Now, Green's theorem will be obtained by using the complete representation given in Eq. (13) with the proper application of the distribution theory and by revealing the differences when compared with Eqs. (II.2.47) and (II.2.48) in Schwartz (2008, p. 88). Using Eq. (13) in Eq. (5) gives

$$
\left\langle\{\Delta V\}+\left(\llbracket\left[\frac{\partial V}{\partial n} \rrbracket-J \llbracket V \rrbracket\right) \delta(S)+\llbracket V \rrbracket \frac{\partial \delta(S)}{\partial n}, t\right\rangle=\langle V, \Delta t\rangle,\right.
$$

and in integral form,

$$
\left.\int\{\Delta V\} t d \vartheta+\int\left(\llbracket \frac{\partial V}{\partial n} \rrbracket\right]-J \llbracket V \rrbracket\right) \delta(S) t d \vartheta+\int \llbracket V \rrbracket \frac{\partial \delta(S)}{\partial n} t d \vartheta=\int V \Delta t d \vartheta
$$

in which the third volume integral on the left must be performed according to Eq. (17), leading to

$$
\begin{gathered}
\int\{\Delta V\} t d \vartheta+\int\left(\llbracket \frac{\partial V}{\partial n} \rrbracket-J \llbracket V \rrbracket\right) t \delta(S) d \vartheta-\int\left(\frac{\partial}{\partial n}(\llbracket V \rrbracket t)-J \llbracket V \rrbracket t\right) \delta(S) d \vartheta= \\
\int V \Delta t d \vartheta
\end{gathered}
$$

where the second and third volume integrals on the left can be replaced by the surface integrals due to $\delta(S)$. For a closed region $\vartheta_{1}=\vartheta$ and its boundary denoted by $\partial \vartheta=S$, $V$ can be chosen to be the function inside $\vartheta$ and be zero outside of it, then $\llbracket V \rrbracket=$ $0-V=-V$ on $S$, which is only a function of surface coordinates. If Eq. (22) is rewritten to

$$
\int_{\vartheta} \Delta V t d \vartheta-\oint_{\partial \vartheta=S}\left(\frac{\partial V}{\partial n}-J V\right) t d S+\oint_{\partial \vartheta=S}\left(V \frac{\partial t}{\partial n}-J V t\right) d S=\int_{\vartheta} V \Delta t d \vartheta
$$

and by rearranging,

$$
\int_{\vartheta}(\Delta V t-V \Delta t) d \vartheta=\oint_{\partial \vartheta=S}\left(\frac{\partial V}{\partial n} t-V \frac{\partial t}{\partial n}\right) d S
$$

is obtained, which is the very well-known form of Green's theorem.

\section{Revised Distributional Forms of the Poisson Equation and Surface Charge Densities}

As mentioned by Van Bladel (1995, p. 22), the Poisson equation

$$
\Delta V=-\rho / \varepsilon_{0}
$$


is valid in the sense of distributions throughout space, where $V$ is the potential, $\rho$ is the volume charge density, and $\varepsilon_{0}$ is the permittivity of free space. If one considers Eq. (5) with Eq. (25), $t=1 / R$, where $R=|\vec{R}|=\left|\vec{r}-\vec{r}^{\prime}\right|$ is the distance from observation point $\vec{r}$ to source point $\vec{r}^{\prime}$, and the Laplacian of $t$ with respect to the primed coordinates, which is $\Delta^{\prime} t=\Delta^{\prime}(1 / R)=\Delta(1 / R)=-4 \pi \delta\left(\vec{r}-\vec{r}^{\prime}\right)$, one can obtain

$$
V(\vec{r})=\frac{1}{4 \pi \varepsilon_{0}} \int \frac{\rho\left(\vec{r}^{\prime}\right)}{R} d \vartheta^{\prime}
$$

By using the distributional form of the Poisson equation, the volume charge density and also the boundary conditions can be obtained in a sense. Therefore, Eq. (13) should be used in Eq. (25) to determine the charge densities and their types in the space to give

$$
\Delta V=\{\Delta V\}+\left(\llbracket \frac{\partial V}{\partial n} \rrbracket-J \llbracket V \rrbracket\right) \delta(S)+\llbracket V \rrbracket \frac{\partial \delta(S)}{\partial n}=-\rho / \varepsilon_{0},
$$

from which one can deduce that the volume charge density is written in the form of

$$
\rho=\{\rho\}+\rho_{0} \delta(S)+\rho_{1} \frac{\partial \delta(S)}{\partial n} .
$$

By comparing Eqs. (27) and (28), one can obtain $\{\rho\}=\left\{-\varepsilon_{0} \Delta V\right\}, \quad \rho_{0}=$ $-\varepsilon_{0}(\llbracket \partial V / \partial n \rrbracket-J \llbracket V \rrbracket)$, and $\rho_{1}=-\varepsilon_{0} \llbracket V \rrbracket$, and, as can be seen, $\llbracket V \rrbracket$ contributes not only to $\delta^{(1)}(S)$ but also to $\delta(S)$, which means that in addition to the double-layer charge density, $\llbracket V \rrbracket \neq 0$ may also cause a single-layer charge density related to $J$, namely the geometric properties of the surface. Since the double layer can be formed in two ways-a dipole layer and an equidensity double layer - the charge representations related to $\delta^{(1)}(S)$ and its combination with $\delta(S)$ are now examined to interpret the result obtained in Eq. (27). Inserting the first term in the right side of Eq. (28) into Eq. (26) gives the potential due to the charges that are not located on the surface $S$ as

$$
V_{\{\}}(\vec{r})=\frac{1}{4 \pi \varepsilon_{0}} \int \frac{\left\{\rho\left(\vec{r}^{\prime}\right)\right\}}{R} d \vartheta^{\prime}
$$

It is known that $\rho_{0} \delta(S)$ represents a single-layer charge distribution, and its potential can also be obtained from Eq. (26) as

$$
V_{0}(\vec{r})=\frac{1}{4 \pi \varepsilon_{0}} \int_{S} \frac{\rho_{0}\left(\vec{r}^{\prime}\right)}{R} d S^{\prime}
$$

Finally, inserting $\rho_{1} \delta^{(1)}(S)$ into Eq. (26) using Eq. (17), it can be seen that the result for the potential function will be

$$
V_{1}(\vec{r})=-\frac{1}{4 \pi \varepsilon_{0}} \int_{S} \rho_{1}\left(\vec{r}^{\prime}\right)\left[\frac{\partial}{\partial n^{\prime}}\left(\frac{1}{R}\right)-\frac{J}{R}\right] d S^{\prime}
$$


which is quite different than the well-known one given as

$$
V_{d i p}(\vec{r})=-\frac{1}{4 \pi \varepsilon_{0}} \int_{S} \rho_{1}\left(\vec{r}^{\prime}\right) \frac{\partial}{\partial n^{\prime}}\left(\frac{1}{R}\right) d S^{\prime}
$$

for a dipole layer (Stratton, 1941, p. 189; Jackson, 1975, p. 37; Van Bladel, 1995, p. 20 and p. 206), which means $\rho_{1} \delta^{(1)}(S)$ cannot be the distributional representation for the volume density of a dipole layer on $S$. Therefore, the correct distributional form of a dipole layer must be obtained. The investigation of equivalent volume charge densities for various types of charge distributions was presented in detail in Namias (1977). By considering the results obtained by Namias (1977) and the divergence in the sense of distributions as in Eq. (2), one can see that the equivalent volume charge density for a distribution of dipoles confined within a volume can also be written in a compact form as

$$
\rho=-\nabla \cdot \vec{P}
$$

like the well-known one for a continuous distribution of dipoles where $\vec{P}$ is the dipole moment density. In addition to that, for a special case of dipoles distributed with a surface density of $P_{S}$ on $S$, the volume density of the dipole moment is written as $\vec{P}=P_{S} \hat{n} \delta(S)$, and the volume charge density can also be obtained in implicit form as

$$
\rho_{\text {dip }}=-\nabla \cdot\left(P_{S} \hat{n} \delta(S)\right)
$$

Now the explicit form of $\rho_{\text {dip }}$ given in Eq. (34) will be obtained by using the identities in Gagnon (1970), and since $P_{S}$ is only a function of surface coordinates,

$$
\begin{aligned}
\rho_{\text {dip }} & =-\left[P_{S} \delta(S) \nabla \cdot \hat{n}+\nabla\left(P_{S} \delta(S)\right) \cdot \hat{n}\right] \\
& =J P_{S} \delta(S)-\frac{\partial}{\partial n}\left(P_{S} \delta(S)\right) \\
& =J P_{S} \delta(S)-P_{S} \frac{\partial \delta(S)}{\partial n}
\end{aligned}
$$

for the distributional form of a dipole layer with not only $-P_{S} \partial \delta(S) / \partial n$ as given in Van Bladel (1995, p. 20). To check its correctness, the potential function can be found by inserting Eq. (35) into Eq. (26) and considering Eq. (17) as

$$
V_{d i p}(\vec{r})=\frac{1}{4 \pi \varepsilon_{0}} \int_{S} P_{S}\left(\vec{r}^{\prime}\right) \frac{\partial}{\partial n^{\prime}}\left(\frac{1}{R}\right) d S^{\prime}
$$

which is the correct form of the potential of a dipole layer as presented in Eq. (32). Therefore, it can be concluded that the revised, correct, and complete form of the distributional representation of a dipole layer is given by Eq. (35). The types of resulting surface charge distributions due to the discontinuity of the potential function can now be determined by considering the distributional form of the Poisson equation given in Eq. (27), and the whole charge distribution is obtained as 


$$
\left.\rho=\left\{-\varepsilon_{0} \Delta V\right\}-\varepsilon_{0} \llbracket \frac{\partial V}{\partial n} \rrbracket\right] \delta(S)+\varepsilon_{0} J \llbracket V \rrbracket \delta(S)-\varepsilon_{0} \llbracket V \rrbracket \frac{\partial \delta(S)}{\partial n},
$$

where $\left\{-\varepsilon_{0} \Delta V\right\}$ corresponds to the volume charges when $\vec{r} \notin S$, the second term in the right side is the distributional form of a single-layer surface charge density $-\varepsilon_{0} \llbracket \partial V / \partial n \rrbracket$ on $S$, and finally, the last two terms in the right side of Eq. (37) are the correct representation of the distributional form of a dipole layer given as

$$
\rho_{\text {dip }}=J \varepsilon_{0} \llbracket V \rrbracket \delta(S)-\varepsilon_{0} \llbracket V \rrbracket \frac{\partial \delta(S)}{\partial n}
$$

due to $\llbracket V \rrbracket \neq 0$, and $\varepsilon_{0} \llbracket V \rrbracket$ corresponds to the surface density of dipoles on $S$. The charge representation of $\partial \delta(S) / \partial n$ by itself will now be discussed. In Van Bladel (1995, p. 206), it was seen that a double layer can be formed in two ways by considering equal total charges or equal charge densities on differential surfaces of two single layers separated by a small distance $h$ and letting $h \rightarrow 0$; those are called the dipole layer or equidensity layer, respectively. With the details given in Van Bladel (1995, p. 207), to obtain an equidensity layer, one must add a single layer of density $-J P_{S}$, in distributional terms $-J P_{S} \delta(S)$, to the dipole layer of Eq. (35), which means

$$
\rho_{\text {equi }}=-P_{S} \frac{\partial \delta(S)}{\partial n}
$$

is the distributional representation for an equidensity layer, and it is also different when compared with the one given in Van Bladel (1995, p. 207). Therefore, one can conclude that

$$
\rho_{e q u i}=-\varepsilon_{0} \llbracket V \rrbracket \frac{\partial \delta(S)}{\partial n}
$$

is the revised and correct distributional form of an equidensity layer due to $\llbracket V \rrbracket \neq 0$. The potential function for an equidensity layer given in Eq. (39) can again be obtained by Eqs. (26) and (17) as

$$
V_{\text {equi }}(\vec{r})=\frac{1}{4 \pi \varepsilon_{0}} \int_{S} P_{S}\left(\vec{r}^{\prime}\right)\left[\frac{\partial}{\partial n^{\prime}}\left(\frac{1}{R}\right)-\frac{J}{R}\right] d S^{\prime} .
$$

Since $R_{1} \rightarrow-\infty$ and $R_{2} \rightarrow-\infty$ for a planar surface, which results in $J=0$, the double layer is a dipole layer and also an equidensity layer in that case; the representations given in Eqs. (35) and (39) are identical, and so are Eqs. (36) and (41). Double-layer examples are now given by the general approach given above for comparison purposes. First considered is the two metal parallel plate problem, the planar case, with a potential difference $V$ between the upper plate located at $z=b=a+h$ and the lower plate located at $z=a$. Using the surface charge densities obtained as $\rho_{S_{b}}=\varepsilon_{0} V / h$ at $z=b$ and $\rho_{S_{a}}=$ $-\varepsilon_{0} V / h$ at $z=a$, the volume charge density is written as $\rho=\rho_{S_{b}} \delta(z-b)+\rho_{S_{a}} \delta(z-a)$ or $\rho=\varepsilon_{0} V[\delta(z-a-h)-\delta(z-a)] / h$. To form a double layer, letting $h \rightarrow 0$ gives $\rho=-\varepsilon_{0} V d \delta(z-a) / d z$. Considering surface $S$ being $z=a$ plane, since $\delta(S)=\delta(z-a)$, $\delta^{(1)}(S)=d \delta(z-a) / d z, J=0$, and $\llbracket V \rrbracket=V$ as $h \rightarrow 0, \rho=-\varepsilon_{0} \llbracket V \rrbracket \delta^{(1)}(S)$ can be written, which is consistent with the explanations given above and the form given in Eqs. (35) 
and (38) with $J=0$, and also Eqs. (39) and (40) where the double layer is a dipole layer and an equidensity layer.

The second example examines two coaxial metal cylinders of radii $p=b=a+h$ and $p=a$ with a potential difference $V$ between them, and instead of $\rho$ generally used to represent the distance from the $z$-axis in the cylindrical coordinates, $p$ is used to prevent confusion with the charge density. Using the surface charge densities obtained as $\rho_{S_{b}}=$ $\varepsilon_{0} V /(b \ln (b / a))$ and $\rho_{S_{a}}=-\varepsilon_{0} V /(a \ln (b / a))$, the volume charge density can be written as $\rho=\rho_{S_{b}} \delta(p-b)+\rho_{S_{a}} \delta(p-a)$ or $\rho=\varepsilon_{0} V[\delta(p-a-h) /(a+h)-\delta(p-a) /$ $a] / \ln (1+h / a)$; after mathematical manipulations and to form a double layer by letting $h \rightarrow 0 \quad$ with $\ln (1+h / a) \cong h / a$ gives $\rho=\varepsilon_{0} V[-d \delta(p-a) / d p-(1 / a) \delta(p-a)]$. Considering $S$ being the cylindrical surface with radius $p=a$, since $\delta(S)=\delta(p-a)$, $\delta^{(1)}(S)=d \delta(p-a) / d p, R_{1}=-a, R_{2} \rightarrow-\infty, J=-1 / a$, and $\llbracket V \rrbracket=V$ as $h \rightarrow 0, \rho=$ $J \varepsilon_{0} \llbracket V \rrbracket \delta(S)-\varepsilon_{0} \llbracket V \rrbracket \delta^{(1)}(S)$ can be written, which is also consistent with the form given in Eqs. (35) and (38), and in this case, the double layer is a dipole layer, as expected.

Finally, two concentric metal spheres are considered, with radii $r=b=a+h$ and $r=a$ with a potential difference $V$ between them, where $r$ is the distance from the origin in spherical coordinates. Since the surface charge densities can be obtained as $\rho_{S_{b}}=$ $\varepsilon_{0} V /\left[(1 / a-1 / b) b^{2}\right]$ and $\rho_{S_{a}}=-\varepsilon_{0} V /\left[(1 / a-1 / b) a^{2}\right]$, the volume charge density can then be written similar to the previous ones as $\rho=\rho_{S_{b}} \delta(r-b)+\rho_{S_{a}} \delta(r-a)$. To form a double layer, letting $h \rightarrow 0$ gives $\rho=\varepsilon_{0} V[-d \delta(r-a) / d r-(2 / a) \delta(r-a)]$. Considering $S$ being the spherical surface with radius $r=a$, since $\delta(S)=\delta(r-a)$, $\delta^{(1)}(S)=d \delta(r-a) / d r, \quad R_{1}=R_{2}=-a, J=-2 / a$, and $\llbracket V \rrbracket=V \quad$ as $h \rightarrow 0, \rho=$ $J \varepsilon_{0} \llbracket V \rrbracket \delta(S)-\varepsilon_{0} \llbracket V \rrbracket \delta^{(1)}(S)$ can be written, which is also consistent with the form given in Eqs. (35) and (38), and in this case, the double layer is again a dipole layer, as expected.

After these examples, the boundary conditions are now examined by considering the Poisson equation given in Eq. (27) with Eq. (28); since $\vec{D}=\varepsilon_{0} \vec{E}=-\varepsilon_{0} \nabla V$ and $\hat{n} \cdot \llbracket \vec{D} \rrbracket=-\varepsilon_{0} \llbracket \partial V / \partial n \rrbracket$,

$$
\hat{n} \cdot \llbracket \vec{D} \rrbracket=-\varepsilon_{0} \llbracket \frac{\partial V}{\partial n} \rrbracket=\rho_{0}-\varepsilon_{0} J \llbracket V \rrbracket
$$

for the discontinuity of the normal component of $\vec{D}$ on $S$. Now for the special case of a dipole layer, charge density is given as $\rho=J \varepsilon_{0} \llbracket V \rrbracket \delta(S)-\varepsilon_{0} \llbracket V \rrbracket \delta^{(1)}(S)$, leading to $\left\{-\varepsilon_{0} \Delta V\right\}=0$ and $-\varepsilon_{0} \llbracket \partial V / \partial n \rrbracket=0$ by Eq. (37), or $\{\rho\}=0, \rho_{0}=\varepsilon_{0} J \llbracket V \rrbracket$, and $\rho_{1}=$ $-\varepsilon_{0} \llbracket V \rrbracket$ in Eq. (28); so in both ways, by Eq. (42), $\hat{n} \cdot[[\vec{D}]]=0$, giving $\llbracket D_{n} \rrbracket=0$ and $\llbracket E_{n} \rrbracket=0$, namely the normal components are continuous across the surface as expected for a dipole layer (Stratton, 1941, p. 191; Van Bladel, 1995, p. 207). For the case of an equidensity layer, charge density is given as $\rho=-\varepsilon_{0} \llbracket V \rrbracket \delta^{(1)}(S)$, leading to $\left\{-\varepsilon_{0} \Delta V\right\}=0$ and $-\varepsilon_{0} \llbracket \partial V / \partial n \rrbracket=-\varepsilon_{0} J \llbracket V \rrbracket$ by Eq. (37), or $\{\rho\}=0, \rho_{0}=0$, and $\rho_{1}=-\varepsilon_{0} \llbracket V \rrbracket$ in Eq. (28); so in both ways, by Eq. (42), $\hat{n} \cdot[[\vec{D}]]=-\varepsilon_{0} J \llbracket V \rrbracket$, giving $\llbracket D_{n} \rrbracket=-\varepsilon_{0} J \llbracket V \rrbracket$ and $\llbracket E_{n} \rrbracket=-J \llbracket V \rrbracket$, namely the normal components are discontinuous across the surface, as expected for an equidensity layer (Van Bladel, 1995, p. 207). For the very special case of a planar equidensity layer, which is also a planar dipole layer, since $J=0, \llbracket D_{n} \rrbracket=0$, and $\llbracket E_{n} \rrbracket=0$, results in continuous normal components across the surface. 


\section{Generalizations for Multi-Layers}

In this section, general expressions are obtained for when there is a multi-layer on the discontinuity surface. Examining the distributional forms presented in the previous sections, it can be seen that for potential function $V=\{V\}$, the electric field is obtained, using Eq. (1), as $\vec{E}=-\nabla V=-(\{\nabla V\}+\hat{n} \llbracket V \rrbracket \delta(S))=\{\vec{E}\}+\vec{E}_{0} \delta(S)$, and the charge density, from Eqs. (27) and (28), as $\rho=\{\rho\}+\rho_{0} \delta(S)+\rho_{1} \delta^{(1)}(S)$, resulting in a double layer on $S$. Considering these results and a theorem that a distribution with compact support has a finite order (Friedlander \& Joshi, 1998; Friedman, 2005; Vladimirov, 2002, p. 36; Polat, 2011), it can be concluded that the general distributional representations for multi-layers can be given as

$$
\rho=\{\rho\}+\sum_{k=0}^{N} \rho_{k} \delta^{(k)}(S)
$$

and

$$
\vec{E}=\{\vec{E}\}+\sum_{k=0}^{N} \vec{E}_{k} \delta^{(k)}(S)
$$

for scalars and vectors, respectively. In Eqs. (43) and (44), \{\} denotes the regular part of the quantity considered as mentioned previously, and the term with the summation represents the singular part of the distribution, which consists of the linear combination of the Dirac delta distribution $\delta(S)$ and its normal derivatives $\delta^{(k)}(S)$ concentrated on the surface of discontinuity. $N$ is the order of the distributions and is finite due to the theorem. For the most general form of the Poisson equation, the Laplacian of a potential function must first be obtained, given in the form of Eq. (43), namely

$$
V=\{V\}+\sum_{k=0}^{N} V_{k} \delta^{(k)}(S)
$$

and since $\Delta V=\nabla \cdot(\nabla V)$, the gradient of $V$ given in Eq. (45) must be obtained, then its divergence found; thus,

$$
\begin{aligned}
\nabla V & =\nabla\left(\{V\}+\sum_{k=0}^{N} V_{k} \delta^{(k)}(S)\right) \\
& =\nabla\{V\}+\nabla\left(\sum_{k=0}^{N} V_{k} \delta^{(k)}(S)\right) \\
& =\{\nabla V\}+\hat{n} \llbracket V \rrbracket \delta(S)+\nabla\left(\sum_{k=0}^{N} V_{k} \delta^{(k)}(S)\right),
\end{aligned}
$$

and for the gradient of the last term in Eq. (46), considering the identities given by Gagnon (1970) and that $V_{k}$ is a function of surface coordinates, then 


$$
\begin{aligned}
\nabla V= & \{\nabla V\}+\left(\hat{n} \llbracket V \rrbracket+\nabla_{S} V_{0}\right) \delta(S) \\
& +\sum_{k=1}^{N}\left(\nabla_{S} V_{k}+\hat{n} V_{k-1}\right) \delta^{(k)}(S) \\
& +\hat{n} V_{N} \delta^{(N+1)}(S)
\end{aligned}
$$

and for a vector $\vec{A}$ given in a general form of Eq. (44), its divergence

$$
\begin{aligned}
\nabla \cdot \vec{A}= & \nabla \cdot\left(\{\vec{A}\}+\sum_{k=0}^{N} \vec{A}_{k} \delta^{(k)}(S)\right) \\
= & \left.\{\nabla \cdot \vec{A}\}+(\hat{n} \cdot \llbracket \vec{A}]+\nabla_{S} \cdot \vec{A}_{0}\right) \delta(S) \\
& +\sum_{k=1}^{N}\left(\nabla_{S} \cdot \vec{A}_{k}+\hat{n} \cdot \vec{A}_{k-1}\right) \delta^{(k)}(S) \\
& +\hat{n} \cdot \vec{A}_{N} \delta^{(N+1)}(S)
\end{aligned}
$$

can be obtained similarly. If the vector obtained in Eq. (47) is called $\vec{A}$, with $\{\vec{A}\}=\{\nabla V\}, \quad \vec{A}_{0}=\hat{n} \llbracket V \rrbracket+\nabla_{S} V_{0}, \vec{A}_{k}=\nabla_{S} V_{k}+\hat{n} V_{k-1} \quad($ for $\quad k=1, \ldots, N), \quad$ and $\vec{A}_{N+1}=\hat{n} V_{N}$, and is inserted into Eq. (48), the following is obtained after lengthy mathematical manipulations:

$$
\begin{aligned}
\Delta V= & \{\Delta V\}+\left(\Delta_{S} V_{0}-J \llbracket V \rrbracket+\llbracket\left\lceil\frac{\partial V}{\partial n} \rrbracket\right) \delta(S)\right. \\
& +\left(\Delta_{S} V_{1}-J V_{0}+\llbracket V \rrbracket\right) \delta^{(1)}(S) \\
& +\sum_{k=2}^{N}\left(\Delta_{S} V_{k}-J V_{k-1}+V_{k-2}\right) \delta^{(k)}(S) \\
& +\left(-J V_{N}+V_{N-1}\right) \delta^{(N+1)}(S) \\
& +V_{N} \delta^{(N+2)}(S)
\end{aligned}
$$

as the general distributional form of the Laplacian of $V$. In Eq. (49), $\Delta_{S}=\nabla_{S} \cdot \nabla_{S}$ is the surface Laplacian of a scalar function as defined and given in Van Bladel (2007, p. 1028). One can see that if $V_{k}=0$ for $k=0,1, \ldots, N$ in Eqs. (45) and (49), Eq. (13) is obtained, as expected. If Eq. (49) is used in Eq. (25), the general distributional form of the Poisson equation is obtain, and it can be concluded that the charge density must be represented as

$$
\rho=-\varepsilon_{0} \Delta V=\{\rho\}+\sum_{k=0}^{N+2} \rho_{k} \delta^{(k)}(S),
$$

where $\quad\{\rho\}=\left\{-\varepsilon_{0} \Delta V\right\}, \quad \rho_{0}=-\varepsilon_{0}\left(\Delta_{S} V_{0}-J \llbracket V \rrbracket+\llbracket \partial V / \partial n \rrbracket\right), \quad \rho_{1}=-\varepsilon_{0}\left(\Delta_{S} V_{1}-\right.$ $\left.J V_{0}+\llbracket V \rrbracket\right)$, and $\rho_{k}=-\varepsilon_{0}\left(\Delta_{S} V_{k}-J V_{k-1}+V_{k-2}\right)$ (for $\left.k=2,3, \ldots, N\right), \rho_{N+1}=$ $-\varepsilon_{0}\left(-J V_{N}+V_{N-1}\right)$ and $\rho_{N+2}=-\varepsilon_{0} V_{N}$ by comparison imply 


$$
\hat{n} \cdot\left[[\vec{D}]=-\varepsilon_{0}\left[\llbracket \frac{\partial V}{\partial n} \rrbracket=\rho_{0}-\varepsilon_{0} J \llbracket V \rrbracket+\varepsilon_{0} \Delta_{S} V_{0}\right.\right.
$$

for the discontinuity of the normal component of $\vec{D}$ on $S$. It is clear that the boundary relation given in Eq. (42) is a special case of the most general one given in Eq. (51). The potential function for a multi-layer can be obtained in terms of a general form of charge density, which is the solution of the generalized distributional form of the Poisson equation, as

$$
\begin{aligned}
V(\vec{r})= & \frac{1}{4 \pi \varepsilon_{0}} \int \frac{\left\{\rho\left(\vec{r}^{\prime}\right)\right\}+\sum_{k=0}^{N} \rho_{k}\left(\vec{r}^{\prime}\right) \delta^{(k)}(S)}{R} d \vartheta^{\prime} \\
= & \frac{1}{4 \pi \varepsilon_{0}} \int \frac{\left\{\rho\left(\vec{r}^{\prime}\right)\right\}}{R} d \vartheta^{\prime}+\frac{1}{4 \pi \varepsilon_{0}} \int_{S} \frac{\rho_{0}\left(\vec{r}^{\prime}\right)}{R} d S^{\prime} \\
& +\frac{1}{4 \pi \varepsilon_{0}} \sum_{k=1}^{N}(-1)^{k} \int_{S} \rho_{k}\left(\vec{r}^{\prime}\right)\left[\left(\frac{\partial}{\partial n^{\prime}}-J\right)^{k} \frac{1}{R}\right] d S^{\prime}
\end{aligned}
$$

by using Eq. (26) with Eq. (18) similar to Eqs. (29)-(31). If the charges are only located on the boundary, the terms with \{\} , and therefore their integrals, vanish in Eq. (52). In case of a region $\vartheta$ bounded by $S$, if $V$ is chosen to be the function inside $\vartheta$ and be zero outside of it, and if the generalized distributional form of the Laplacian $\Delta V$ is known, one can obtain the multi-layer potential at any interior point as

$$
\begin{aligned}
V(\vec{r})= & -\frac{1}{4 \pi} \int_{\vartheta} \frac{\Delta^{\prime} V}{R} d \vartheta^{\prime}-\frac{1}{4 \pi} \oint_{\partial \vartheta=S}\left(\frac{\Delta_{S}^{\prime} V_{0}}{R}-\frac{1}{R} \frac{\partial V}{\partial n^{\prime}}+V \frac{\partial}{\partial n^{\prime}}\left(\frac{1}{R}\right)\right) d S^{\prime} \\
& +\frac{1}{4 \pi} \oint_{\partial \vartheta=S}\left(\Delta_{S}^{\prime} V_{1}-J V_{0}\right)\left[\frac{\partial}{\partial n^{\prime}}\left(\frac{1}{R}\right)-\frac{J}{R}\right] d S^{\prime} \\
& +\frac{1}{4 \pi} \sum_{k=2}^{N}(-1)^{k+1} \oint_{\partial \vartheta=S}\left(\Delta_{S}^{\prime} V_{k}-J V_{k-1}+V_{k-2}\right)\left[\left(\frac{\partial}{\partial n^{\prime}}-J\right)^{k} \frac{1}{R}\right] d S^{\prime} \\
& +\frac{(-1)^{N+2}}{4 \pi} \oint_{\partial \vartheta=S}\left(-J V_{N}+V_{N-1}\right)\left[\left(\frac{\partial}{\partial n^{\prime}}-J\right)^{N+1} \frac{1}{R}\right] d S^{\prime} \\
& +\frac{(-1)^{N+3}}{4 \pi} \oint_{\partial \vartheta=S} V_{N}\left[\left(\frac{\partial}{\partial n^{\prime}}-J\right)^{N+2} \frac{1}{R}\right] d S^{\prime},
\end{aligned}
$$

and if $V$ is harmonic in the region $\vartheta$, then $\Delta V=0$, corresponding to the case of no charge in $\vartheta$, resulting in the disappearance of the first term in the right-hand side of Eq. (53), which means the potential can be obtained by using the values of $V, \partial V / \partial n, V_{k}$, and $\Delta_{S} V_{k}$ over $S$. As a result, that form of Eq. (53) can be interpreted as the solution of the Laplace equation in region $\vartheta$, satisfying the specified boundary conditions when there is a multi-layer on surface $S$. The above version of potential $V$ in Eq. (53) can also be obtained from the most general form of Green's theorem. Therefore, finally, the generalized Green's 
theorem is obtained in region $\vartheta$ bounded by $S$ when there is a multi-layer on the boundary. By following similar steps presented in the second section by using the general distributional form of the Laplacian presented in Eq. (49), and inserting it in Eq. (5) after the necessary mathematical manipulations, and by using Eq. (18), the generalized Green's theorem can be obtained for a multi-layer as

$$
\begin{aligned}
\int_{\vartheta}(\Delta V t-V \Delta t) d \vartheta= & \oint_{\partial \vartheta=S}\left(\frac{\partial V}{\partial n} t-V \frac{\partial t}{\partial n}-\Delta_{S} V_{0} t\right) d S \\
& +\oint_{\partial \vartheta=S}\left(\Delta_{S} V_{1}-J V_{0}\right)\left(\frac{\partial t}{\partial n}-J t\right) d S \\
& +\sum_{k=2}^{N}(-1)^{k+1} \oint_{\partial \vartheta=S}\left(\Delta_{S} V_{k}-J V_{k-1}+V_{k-2}\right)\left[\left(\frac{\partial}{\partial n}-J\right)^{k} t\right] d S \\
& +(-1)^{N+2} \oint_{\partial \vartheta=S}\left(-J V_{N}+V_{N-1}\right)\left[\left(\frac{\partial}{\partial n}-J\right)^{N+1} t\right] d S \\
& +(-1)^{N+3} \oint_{\partial \vartheta=S} V_{N}\left[\left(\frac{\partial}{\partial n}-J\right)^{N+2} t\right] d S .
\end{aligned}
$$

To obtain the potential function given in Eq. (53), it is enough to consider the most general form of Green's theorem presented in Eq. (54) in primed coordinates, $t=1 / R$, where $R=|\vec{R}|=\left|\vec{r}-\vec{r}^{\prime}\right|$, and $\Delta^{\prime} t=\Delta^{\prime}(1 / R)=\Delta(1 / R)=-4 \pi \delta\left(\vec{r}-\vec{r}^{\prime}\right)$, as presented in the previous section.

\section{Conclusion}

In this article, it has been shown that the distributional form for the Laplacian given by Schwartz (2008) and others is incomplete, and one main distributional relation and its usage, and as a result its related usages, are incorrect, leading to wrong results and interpretations mathematically and, hence, electromagnetically. Therefore, the distributional forms of the Laplacian, Poisson's equation, both of the double layers - determined as the dipole layer and equidensity layer - and their boundary relations have been investigated in detail. Their revised and correct forms have been obtained and presented by pointing out the related important points and proper application of the distribution theory by comparisons with those previously presented in the literature. All of the generalized forms, including that of Green's theorem, have been obtained when there is a multi-layer on the surface.

\section{References}

Friedlander, F. G., \& M. Joshi. 1998. Introduction to the theory of distributions, Chap. 3. Cambridge: Cambridge University Press.

Friedman, A. 2005. Generalized functions and partial differential equations, Chap. 3. New York: Dover.

Gagnon, R. J. 1970. Distribution theory of vector fields. Am. J. Phys. 38:879-891. 
İdemen, M. 1973. The Maxwell equations in the sense of distributions. IEEE Trans. Antennas Propagat. 21:736-738.

İdemen, M. 1990. Universal boundary relations of the electromagnetic field. J. Phys. Soc. Japan 59:71-80.

İdemen, M. 2011. Discontinuities in the electromagnetic field. New York: IEEE Press.

Jackson, J. D. 1975. Classical electrodynamics, Chap. 1. New York: John Wiley \& Sons.

Kanwal, R. P. 1983. Generalized functions. New York: Academic Press.

Kanwal, R. P. 1988. A differential geometric solution of a problem in electrostatics. SIAM Rev. 30:301.

Maxwell, J. C. 1954. A treatise on electricity and magnetism. New York: Dover.

Namias, V. 1977. Application of the Dirac delta function to electric charge and multipole distributions. Am. J. Phys. 45:624-630.

Pappas, R. C. 1986. Differential-geometric solution of a problem in electrostatics. SIAM Rev. 28:225-227.

Polat, B. 2005. Remarks on the fundamental postulates on field singularities in electromagnetic theory. IEEE Antennas Propagat. Mag. 47:47-54.

Polat, B. 2006. A note regarding "Remarks on the fundamental postulates on field singularities in electromagnetic theory." IEEE Antennas Propagat. Mag. 48:105.

Polat, B. 2011. Approximate boundary relations on anisotropic sheets. PIER B 29:355-392.

Rădulet, R., \& I. R. Ciric. 1971. Generalized functions in the theory of fields. Rev. Roum. Sci. Techn. Électrotechn. et Énerg. 16:565-591.

Schwartz, L. 1950. Théorie des Distributions. Paris: Hermann.

Schwartz, L. 2008. Mathematics for the physical sciences, Chap. 2. New York: Dover (English translation of Méthodes mathématiques pour les sciences physiques [Schwartz, L. 1965, Paris: Hermann]).

Stratton, J. A. 1941. Electromagnetic theory, Chap. 3. New York: McGraw-Hill.

Tai, C. T. 1992. Generalized vector and dyadic analysis. New York: IEEE Press.

Taylor, J. G. 1956. Classical electrodynamics as a distribution theory. Math. Proc. Cambridge Phil. Soc. 52:119-134.

Taylor, J. G. 1958. Classical electrodynamics as a distribution theory. II. Math. Proc. Cambridge Phil. Soc. 54:258-264.

Van Bladel, J. 1995. Singular electromagnetic fields and sources. New York: IEEE Press.

Van Bladel, J. 1999. On the distributional form of the curl. Microw. Opt. Technol. Lett. 23:97-100.

Van Bladel, J. 2007. Electromagnetic fields. New York: IEEE Press.

Vladimirov, V. S. 2002. Methods of the theory of generalized functions, Chap. 1. London: Taylor \& Francis.

Weatherburn, C. E. 1955. Differential geometry of three dimensions, Chap. 12. Cambridge: Cambridge University Press. 